\section{$\underset{\text { hommes }}{\text { \& migrations }}$}

\section{Hommes \& migrations}

Revue française de référence sur les dynamiques

migratoires

$1304 \mid 2013$

Frontières

\title{
Le Poniente almeriense (Almeria)
}

Contradictions et tensions entre local, national et international sur une zone frontalière

\section{Thibaud de Fortescu}

\section{(2) OpenEdition}

\section{Journals}

Édition électronique

URL : http://journals.openedition.org/hommesmigrations/2654

DOI : 10.4000/hommesmigrations.2654

ISSN : 2262-3353

Éditeur

Musée national de l'histoire de l'immigration

Édition imprimée

Date de publication : 1 octobre 2013

Pagination : 111-118

ISBN : 978-2-919040-24-7

ISSN : $1142-852 X$

Référence électronique

Thibaud de Fortescu, «Le Poniente almeriense (Almeria) », Hommes \& migrations [En ligne], 1304 | 2013, mis en ligne le 01 janvier 2017, consulté le 08 janvier 2020. URL : http://journals.openedition.org/ hommesmigrations/2654; DOI : 10.4000/hommesmigrations.2654 


\section{Motril, Andalousie, 10 septembre 2013}

๑ Manuel Bejar, Ideal (quotidien d'Andalousie) 


\title{
LE PONIENTE ALMERIENSE (ALMERIA) CONTRADICTIONS ET TENSIONS ENTRE LOCAL, NATIONAL ET INTERNATIONAL SUR UNE ZONE FRONTALIĖRE
}

\author{
par THIBAUD DE FORTESCU, doctorant au Centre de recherches et d'analyses \\ géopolitiques, université Paris-VIII.
}

\author{
Le sud de l'Espagne cristallise les problématiques \\ contemporaines du contrôle des frontières de l'Europe. \\ Si au niveau international domine la figure invasive \\ de vagues de migrants clandestins arrivant sur des barques, \\ que relaient les médias espagnols, il en va tout autrement \\ du point de vue local. La province d'Almeria doit son \\ développement économique à une agriculture intensive \\ aujourd'hui dépendante de l'immigration, c'est-à-dire d'une \\ main-d'œuvre bon marché et corvéable à merci.
}

\section{Des politiques de contrôle espagnoles sous la pression de I'UE}

Depuis le début des années 2000, l'immigration est devenue une question importante au sein de la société espagnole. Travaux de recherche, articles de journaux, documentaires... la thématique a été utilisée comme sujet de reportage à de nombreuses reprises. L’Andalousie, séparée de seulement quatorze kilomètres du Maroc, fait figure de frontière sud de l'Espagne et de l'Union européenne. À ce titre, elle est à la fois une zone convoitée par les migrants et protégée par les pouvoirs espagnols et européens car perçue comme un point de passage. Le Poniente almeriense, territoire agro-industriel situé dans la province d'Almeria, apparaît comme un espace clé dans cette transition entre l'Afrique et l'Union européenne, comme celui illustrant le mieux certaines contradictions entre échelle locale, nationale et internationale. En 1997, le gouvernement de José Maria Aznar lança le Plan Sur visant à renforcer le contrôle de la zone frontalière et à freiner l'immigration clandestine. Ces objectifs ont été au cœur des différentes réformes législatives sur l’immigration produites au début des années 2000 . En 1998, le même gouvernement a mis en place le Système intégré de vigilance extérieure (SIVE), dont la gestion revient à la Guardia Civil et qui est effectif depuis 2002. Le système est constitué de différentes stations situées sur le littoral qui permettent de détecter une embarcation au large et 
d'envoyer aussi vite que possible une équipe pour l'intercepter. La mise en place du SIVE s'inscrit dans une dynamique européenne de blindage de la frontière. Le traité d'Amsterdam de 1997 et le Conseil européen de Tampere de 1999 ont posé le ciment d'une politique européenne de l'immigration dans laquelle l'axe sécuritaire jouait un rôle prépondérant. Le 5 novembre 2004, le Conseil de l'Europe approuva le programme de La Haye pour la période 2005-2009 avec comme critère principal de "garantir la sécurité et la justice

Les routes ont donc évolué depuis la fin des années 1990,

sous l'influence de la mise en place des dispositifs de contrôle de la frontière, mais les migrations n'ont pas disparu. Par ailleurs, elles continuent de véhiculer des représentations péjoratives. pour faire face aux nouveaux risques, à travers des actions à dimension européenne ${ }^{1 "}$. L'Espagne a largement contribué à installer la thématique du contrôle des frontières à l'agenda européen, notamment à travers ses contributions aux Conseils européens de Tampere et Séville $(2002)^{2}$. De manière générale, depuis le début des années 2000, l'Espagne a constamment cherché à mettre le sujet au cœur des discussions européennes ${ }^{3}$ tout en ayant une attitude très ambiguë. En effet, devant les réactions les plus négatives en Espagne au moment de la mise en place du SIVE, le gouvernement se défaussa en expliquant que si ce type de dispositif était mis en place, c'était avant tout pour répondre aux exigences européennes. En 2012, le ministère de l'Intérieur - dont le budget était en hausse - consacrait 3,9 millions d'euros à l'entretien et à l'amélioration du SIVE ${ }^{4}$. L'année suivante, 1,5 million est consacré à l'amélioration des stations. Malgré ces efforts et ces investissements, et bien que le système bénéficie de technologies récentes, la frontière n'en est pas pour autant étanche.

\section{Un ensemble de dispositifs qui n'empêchent pas l'existence de migrations irrégulières}

La première embarcation en provenance du Maroc, reconnue médiatiquement et débarquant sur les côtes andalouses, s'est échouée à Tarifa le $1^{\text {er }}$ novembre 1988. Tout au long des années 1990, le phénomène a pris de l'importance, suscitant de nombreuses préoccupations. C'est surtout à la fin des années 1990 et au début des années 2000 qu'il est devenu majeur, à la fois médiatiquement et politiquement. En 2006, une hausse spectaculaire des migrations illégales par pateras ${ }^{5}$ 'est produite en Espagne. Ce phénomène a fait alors la Une des journaux papier et télévisés et l'on s'est mis à parler d'une "crise des cayucos", d'une "avalanche de pateras" aux Canaries et en Andalousie. Ces migrations clandestines étaient alors très intenses et ont fini par conduire à une saturation de certains services et dispositifs. Les centres de rétention ne pouvant pas accueillir tous les migrants - et la loi leur interdisant de garder un migrant plus de quarante jours -, un certain nombre d'immigrés en situation irrégulière ont été relâchés (certains étant transférés d'un centre des Canaries à un centre d'Andalousie avant d'être relâchés). C'est surtout parce que les Canaries recevaient énormément de migrants illégaux - majoritairement en provenance du Maroc, du Sénégal ou de Mauritanie - que la situation était si critique. En effet, les migrations en direction de la péninsule (donc principalement de l'Andalousie) sont plus régulières : les variations entre 2002 et 2009 sont relativement faibles et il y a eu moins de migrants interceptés en 2006 vers l'Espagne péninsulaire qu'en 2001 ou 2003.

\footnotetext{
1. Ruth Ferrero, "La UE como actor en las politicas de inmigracion de los estados miembros ? Una aproximacion al escenario euromediterraneo", in Ricard Zapata-Barrero, Gemma Pinyol (dir.), Los gestores del proceso de inmigracion, Actores y redes en España y Europa, Barcelone, Edicions Bellaterra, 2008.

2. Juan David Sempere Souvannavong, "El blindaje y externalización de la frontera sur", in Garcia Castaño, Francisco Javier, Nina Kressova (dir.), Actas del I Congreso Internacional sobre Migraciones en Andalucía, Grenade, 2011.

3. "Aznar ofrece a Marruecos inversiones para frenar la inmigración ilegal”, in El País, 8 mai 2000 ; “España propondrá a la UE un paquete de medidas para frenar la inmigración ilegal", in El País, 20 mai 2002

4. http://www.lamoncloa.gob.es/ServiciosdePrensa/NotasPrensa/MIR/2012/170412Presupuestolnterior.htm.

5. Terme désignant les embarcations de fortune sur lesquelles les migrants tentent de traverser la Méditerranée.
} 


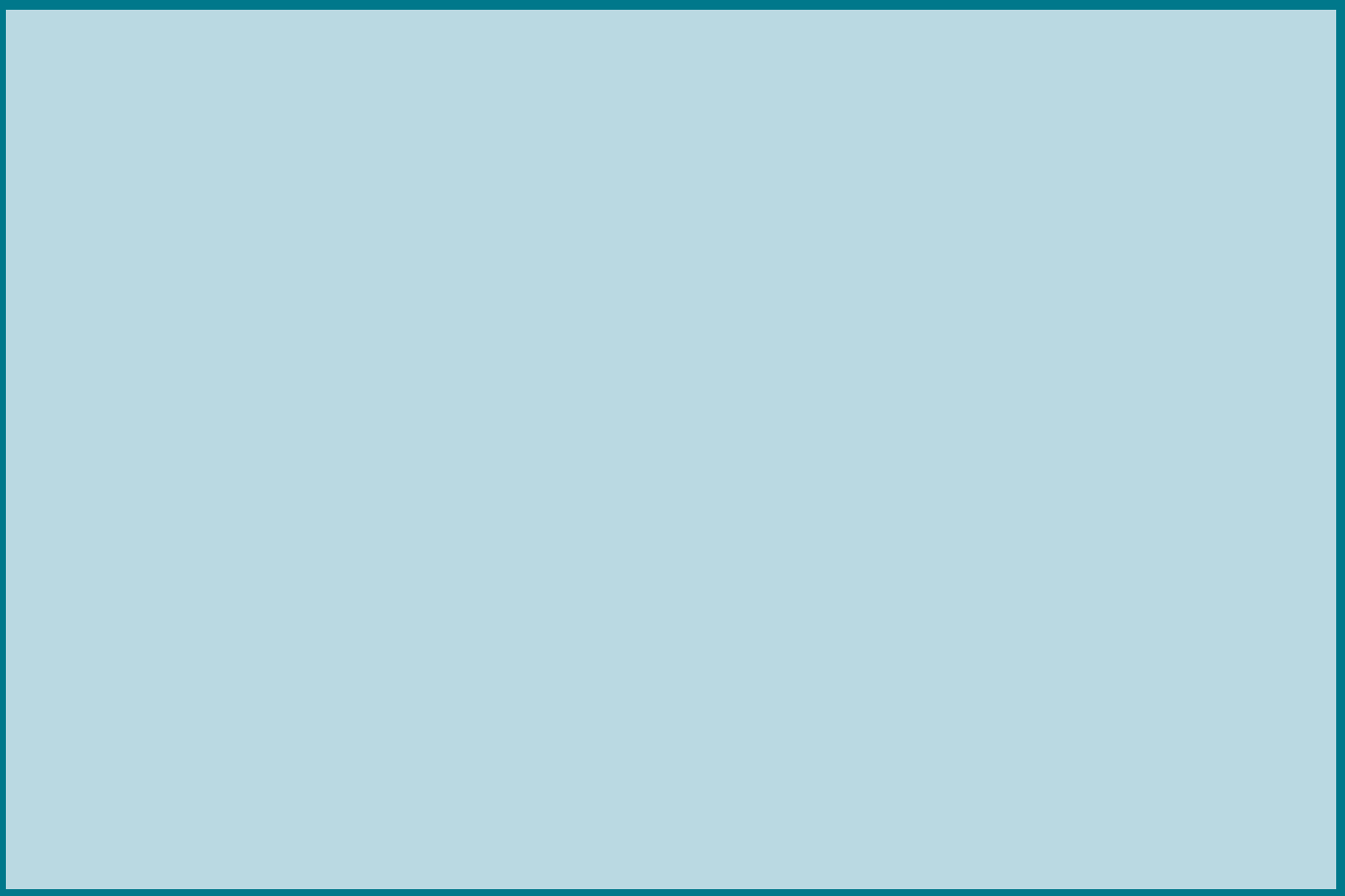

Sources : Élaboration personnelle à partir de la presse (ABC, El Mundo, El Pais, Europa press).

En 2011, on constate que les interceptions de migrants irréguliers concernaient surtout les provinces de Grenade et d'Almeria, soit au large de celles-ci, soit lors de l'accostage des embarcations sur les côtes (figure $\mathrm{n}^{\circ} 1$ ). À elles deux, ces provinces représentaient cette année-là plus de $82 \%$ des migrants et $65 \%$ des embarcations ayant tenté de rejoindre les côtes andalouses. Or, en 1999, ces deux provinces ne représentaient que $27 \%$ des embarcations.

Les routes ont donc évolué depuis la fin des années 1990, sous l'influence de la mise en place des dispositifs de contrôle de la frontière, mais les migrations n'ont pas disparu. Par ailleurs, elles continuent de véhiculer des représentations péjoratives.

\section{Une immigration qui suscite des représentations négatives...}

Bien que le phénomène de l'immigration clandestine ait toujours été marginal, il s'est constamment renforcé sur le plan médiatique jusqu'à devenir incontournable dans les médias. La question spécifique de l'immigration irrégulière et des pateras a joué et continue de jouer un rôle important, contribuant à (dé)former la représentation du phénomène migratoire. C'est en 2000 que la question de l'immigration - au sens large - est apparue dans les enquêtes d'opinion mensuelles du Centro de Investigaciones Sociológicas (CIS, Centre de recherches sociologiques) comme l'un des "princi- 
paux problèmes de l'Espagne". Les arrivées de pateras ont contribué à nourrir la vision de l'immigration comme un "problème", ce dont témoignent les augmentations récurrentes dans ces enquêtes d'opinion au printemps et à l'été 2002, ainsi qu'à la fin des années 2005 et 2006 (périodes correspondant à des hausses des arrivées de pateras).

La résonance des problématiques de l'immigration irrégulière se nourrit de l'important traitement médiatique. Cela est particulièrement vrai pour la presse régionale andalouse, où chaque arrivée ou interception de patera fait l'objet d'un article, mais l'immigration clandestine rencontre également un écho important au niveau national dans l'ensemble des journaux et télévisions. Les représentations que porte cette immigration sont bien singulières. Les pateras, souvent interceptées au large car détectées par des radars, sont remplies de migrants parfois dans un état sanitaire et psychologique précaire. Les traversées peuvent se transformer en calvaire lorsque les conditions météorologiques sont mau-

Bien qu'elle fasse l'objet de violents débats et de multiples dispositifs de contrôle à l'échelle nationale et internationale,

l'immigration, régulière ou irrégulière, nourrit certains secteurs de l'économie espagnole. vaises et que l'embarcation est en mauvais état et/ou trop chargée. L'image renvoyée par les médias de ces débarquements en Andalousie est celle d'une immigration pauvre, voire miséreuse, bien que ce ne soit pas nécessairement les populations les plus pauvres de ces pays qui tentent le voyage. Les photos qui paraissent dans la presse et montrent des sauveteurs ou policiers équipés de masques répandent l'idée que cette immigration peut aussi être le vecteur de maladies et, donc, dangereuse.

Par conséquent, toute l'immigration africaine (maghrébine et noire) a tendance à être assimilée à celle débarquant par pateras tant l'impact médiatique est important. Cela contribue à alimenter les représentations négatives de ces populations qui peuvent être liées à d'autres questions (comme la tendance à la stigmatisation des musulmans en Europe occidentale qui rejaillit sur les Marocains vivant en Espagne).

\section{...et alimente les débats et politiques nationales}

Si le rôle de la presse est important, la mise en avant de l'immigration irrégulière comme "problème national” a été renforcée par les débats politiques et politiciens qui se sont très souvent nourris de cette question.

En juin 2001, José Luis Zapatero, alors leader de l'opposition, accusait le gouvernement Aznar d'avoir "échoué sur le contrôle des frontières", illustrant le fait que le Parti socialiste espagnol pouvait aussi se positionner sur la problématique du contrôle des frontières. Le député du Parti populaire pour Cadix (province frontalière), Jorge Ramos, déclara en juillet de la même année lors d'une session au Parlement que "le PSOE paierait l'essence des pateras s'il le pouvait" en réponse à des critiques socialistes envers la politique du gouvernement, accusé d'empêcher les migrants de venir de manière légale.

Par ailleurs, depuis la fin de la dictature franquiste, l'immigration irrégulière a été au centre des réformes législatives espagnoles sur l'immigration. La (première) loi organique du $1^{\text {er }}$ juillet $1985^{\circ}$ sur les droits et libertés des étrangers en Espagne - dont la majorité des articles sont restés en vigueur jusquà la réforme de 2000 - ordonnait un strict contrôle des entrées. En effet, pour accéder au territoire espagnol, il fallait, entre autres, avoir obtenu un visa dans le pays d'origine et disposer "de moyens économiques suffisants". Des sanctions importantes étaient également prévues pour les situations irrégulières (détention et pri- 
vation de liberté en centre d'internement jusqu'à quarante jours). Cette législation stricte répondait à une volonté de l'Espagne de donner des garanties à l'Union européenne un an avant de l'intégrer, en montrant qu'elle ne constituerait pas une porte d'entrée pour de nombreux clandestins. Rappelons qu'une grande partie des pays de l'Union européenne (dont la France et l'Allemagne) avaient officiellement fermé leurs frontières depuis 1973 et que l'intégration de l'Espagne à l'UE soulevait donc pour ces pays des questions importantes en matière de politique migratoire. Bien qu'elle fasse l'objet de violents débats et de multiples dispositifs de contrôle à l'échelle nationale et internationale, l'immigration, régulière ou irrégulière, nourrit certains secteurs de l'économie espagnole.

\section{L'immigration, une main-d'œuvre indispensable pour le Poniente almeriense}

Aujourd'hui $20 \%$ des actifs de la province d'Almeria travaillent dans l'agriculture contre moins de $5 \%$ sur l'ensemble de l'Espagne. En décembre 2012, dans la province d'Almería, sur les 39164 personnes déclarées au régime agricole, seules 16552 étaient espagnoles (ministère de l'Emploi et de la Sécurité sociale). L'agriculture est le premier secteur d'activité à El Ejido, Vicar et La Mojonera, avec respectivement $41 \%, 40 \%$ et $63 \%$ des actifs occupés.

En 2008, près de $70 \%$ des contrats passés dans le secteur agricole de la province d'Almeria (41 266) concernaient des étrangers (Ministère de l'Emploi ${ }^{10}$. Trois ans plus tard, en 2011, les chiffres restaient proches (72\% et 43103 contrats signés par des étrangers), signe que l'agriculture résistait relativement bien à la crise économique. En 2011, le

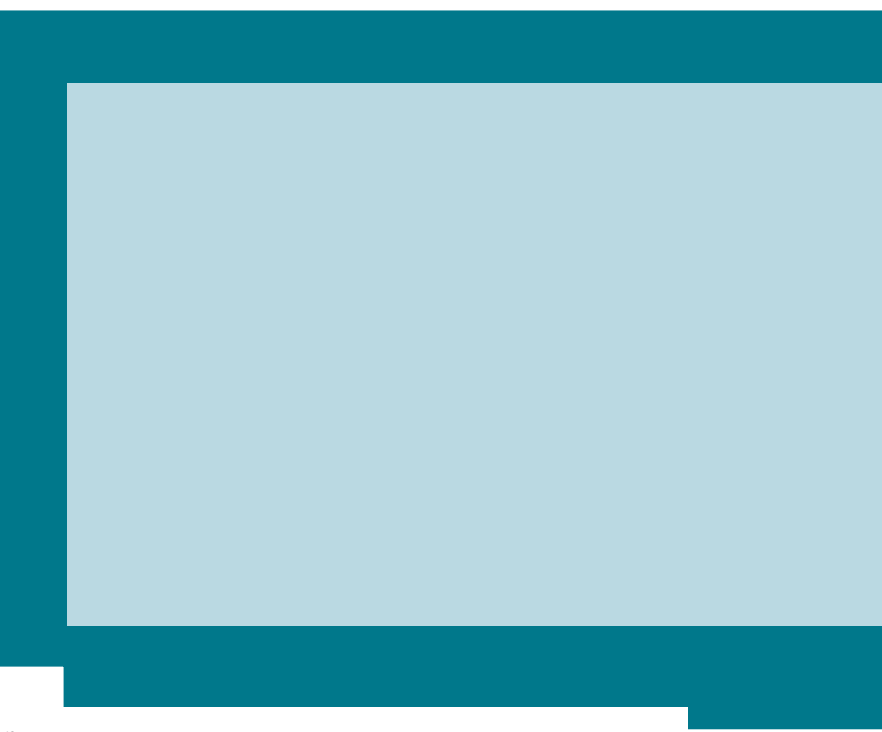

Motril, Andalousie, 10 septembre 2013 (c) Manuel Bejar, Ideal (ouotidien d'Andalousie)

secteur concentrait 54,3\% des contrats signés par des travailleurs étrangers de la province - chiffre qui serait sans doute plus élevé si l'on ne prenait en compte que les étrangers hors Europe de l'Ouest. La segmentation apparaît encore davantage lorsqu'on se concentre sur les emplois d'ouvriers agricoles (76 \% étaient étrangers en 2011, contre $71 \%$ en 2008) ${ }^{11}$.

En décembre 2012, sur l'ensemble de la province, pour un total de 18241 Africains affiliés à la Sécurité sociale, 14430 l'étaient au régime agricole (soit $79 \%$ du total) et les emplois agricoles occupés par les étrangers dans la province sont avant tout ceux d'ouvriers agricoles. Précisons que ces emplois consistent principalement à récolter des fruits ou légumes, à entretenir des plants, à porter des cagettes très lourdes, tout cela sous une chaleur souvent étouffante. Lété, la température dépasse facilement les 40 degrés sous les serres, ce qui pose un certain nombre de problèmes de santé, notamment pour les travailleurs souffrant de pathologies respiratoires ou cardiaques et qui se gardent bien de le signaler, de peur de perdre leur 
emploi. Il est difficile pour ces immigrés - en particulier africains - d'avoir accès à un emploi autre qu'agricole. Sur le terrain, il est aisé de constater à chaque visite d'exploitation agricole ou presque que les travailleurs présents sont des immigrés (marocains, subsahariens ou roumains). Il est très rare de rencontrer un employé d'administration, un vendeur dans un commerce (non ethnique) ou un serveur dans un restaurant qui soit africain. $\grave{A}$ l'inverse, les Roumains ont une meilleure insertion dans l'hôtellerie, les transports, les commercesbars et les restaurants.

Cela ne signifie pas qu'il n'y ait pas de Roumains employés dans l'agriculture, mais ces derniers ne semblent pas rencontrer les mêmes situations de précarité.

\section{L'agriculture, secteur clé de l'économie et de la société locale}

Au début du XX siècle, le Poniente almeriense était pourtant un territoire relégué et abandonné. Les habitants quittaient le territoire pour d'autres villes d'Espagne ou pour l'étranger, et ce jusqu'aux années 1970-1980. Dans les années 1940-1950, une politique de développement lancée par le pouvoir franquiste modifia la situation. Le 24 juin 1941 fut approuvé le décret qui délimita une zone de 30347 hectares ${ }^{12}$ et qui correspond aujourd'hui à peu près à ce que nous appelons le "Poniente almeriense". L'ob- 
jectif était de donner une utilité à ces terres et d'éviter l'exode rural. Un décollage se produisit dans les années 1960 et la politique finit par attirer de nombreux migrants espagnols en provenance des Alpujarras, région montagneuse située entre Grenade et Almeria. Ils trouvèrent là une perspective d'avenir face à la crise de l'agriculture traditionnelle. Les cultures sous serres se développèrent : alors qu'au début des années 1960 il n'y en avait que très peu, en 1984 on en comptait déjà 14000 hectares. Cette évolution de l'agriculture a été accompagnée d'une transformation du territoire. Alors que le Poniente ne comptait que 43516 habitants en 1950, ceux-ci dépassaient 250000 en 2011. Les communes d'El Ejido et La Mojonera ont vu le jour au début des années 1980. L'agriculture au XXIe siècle n'a cependant plus rien à voir avec celle qu'ont connu les premiers migrants espagnols et les habitants originaires de la zone. La production de fruits et de légumes (tomates, melons, poivrons, courgettes, etc.) inonde le marché nord-européen toute l’année et la société s'est transformée. Les agriculteurs du Poniente se sont rapidement enrichis, et dans des proportions importantes.

Entre 1955 et 1975, la province d'Almeria est passée du dernier rang au premier au sein de l'Andalousie en termes de rente agraire. Celle-ci fut multipliée par plus de deux entre 1976 et 1981 . En 1955, le PIB moyen per capita dans la province était de 7809 pesetas (14 586 pour l'Espagne). Dix ans plus tard, il était de 25613 (un peu moins de 154 euros) et, en 1975, de 125572 (un peu plus de 754 euros) $)^{13}$.

Au fil des années et de son développement, le Poniente est devenu une terre prospère vantée comme un modèle de développement, où l'on vient et où l'on reste. Alors qu'en 1995 la province d'Almeria se situait à peu près au niveau moyen de la communauté autonome andalouse, la progression du PIB per capita y a été plus rapide et plus forte qu'ailleurs jusqu'à 2008 (figure $n^{\circ} 2$ 2). Or ces années coïncident avec l'arrivée de nombreuses populations étrangères (principalement marocai- nes puis roumaines) dans la province pour servir de main-d'œuvre à une agriculture délaissée par les Espagnols. Le Poniente almeriense n'a en effet pas échappé à la dynamique nationale, celle du boom économique qu'a connu l'Espagne depuis la deuxième partie des années 1990. Les enfants nés à la fin des années 1970 ou dans les années 1980 ont eu la possibilité de faire des études supérieures, les femmes se sont plus facilement insérées sur le marché de l'emploi, et l'agriculture est devenue un secteur d'emploi moins attractif. Aujourd'hui, des villes du Poniente comme El Ejido, Roquetas de Mar ou La Mojonera comptent plus de $30 \%$ d'immigrés.

\section{Les immigrés, proies idéales d'un secteur informel et opaque}

L'agriculture demeure à ce jour l'un des rares secteurs qui continue d'offrir des opportunités d'emploi à peu près équivalentes à celles d'avant la crise économique. Cependant, il s'agit d'un secteur opaque peu regardant sur le statut des travailleurs. De ce fait, l'agriculture intensive du Poniente représente le secteur idéal pour les immigrés, celui par lequel passe l'espoir d'un emploi qui permettrait de rejoindre d'autres villes ou pays lorsqu'on est en situation régulière - ou d’obtenir une régularisation de sa situation - lorsqu'on est Il y a donc un double processus : les immigrés - et encore davantage pour les plus précaires d'entre eux - sollicitent des emplois agricoles, et l'agriculture se nourrit de cette main-d'œuvre prête à accepter beaucoup de choses pour obtenir un emploi et un meilleur statut. en situation irrégulière. Il y a donc un double processus : les immigrés et encore davantage pour les plus précaires d'entre eux - sollicitent des emplois agricoles, et l'agriculture se nourrit de cette main-d'œuvre prête à accepter beaucoup de choses pour obtenir un emploi et un meilleur statut. 
Pour être précis, ajoutons qu'il existe en fait deux principaux types d'abus : celui qui consiste en l'emploi d'un travailleur en situation irrégulière qui sera donc quasi systématiquement sous-payé voire non payé, et celui de l'emploi d'un travailleur en situation régulière mais dont le salaire n'atteindra pas le minimum légal (5,60 euros/heure selon la convention collective de 2012).

Ces abus sont largement niés par une grande partie des acteurs locaux : élus, organisations patronales, institutions ou même journalistes. Les nombreux contrôles - supposés - de l'Inspection du travail sont mis en avant par les différents interlocuteurs pour expliquer l'impossibilité pour les entrepreneurs d'employer des immigrés en situation irrégulière. Pour ces derniers, il n'est pas envisageable de refuser un emploi, aussi précaire soit-il. En effet, l'accumulation de travailleurs immigrés au sein du Poniente fait qu'en cas de refus, l'employeur n'aura aucun problème pour trouver un autre employé. C'est ce mécanisme qui permet aux agriculteurs de sous-payer un travailleur en situation régulière. La forte présence de main-d'œuvre sur le marché du travail offre la possibilité d'une mise en concurrence des travailleurs et exerce une pression venue de l'extérieur de la structure entrepreneuriale. Qui plus est, sur le terrain, nombreux sont les témoignages qui affirment que l'Inspection prévient les entrepreneurs avant d'effectuer des contrôles, ce qui permet à ces derniers d'éviter tout problème. La responsabilité de l'État espagnol, dont dépend l'Inspection du travail, est à ce titre largement engagée car cela contribue à maintenir la forte précarité que subissent de nombreux travailleurs du secteur, et amplifie les barrières pour une meilleure intégration sociale de ces derniers.

Dans cette perspective, il s'agit de rappeler qu'il n'existe pas dans le Poniente de famille n'ayant pas un de ses membres ou un ami travaillant de près ou de loin dans l'agriculture. Outre le fait que cela a des conséquences directes pour les immigrés - dès qu'un travailleur pâtit d'une mauvaise réputation auprès d'un entrepreneur, il peut rapidement avoir des difficultés pour (re)trouver un emploi -, rappelons que cette activité représente bien plus que de simples emplois.

Le poids que revêt l'agriculture est tel que les liens avec le pouvoir politique sont très forts. De par son importance dans l'économie mais plus largement dans la société locale et dans les représentations collectives, les liens entre les milieux politiques et agricoles se sont largement développés.

De nombreux élus du Poniente sont directement issus de ce milieu, ayant travaillé dans des entreprises du secteur ou ayant été agriculteurs euxmêmes. Le cas le plus représentatif est celui de Gabriel Amat, maire de Roquetas de Mar (Parti Populaire), ancien agriculteur. Né à Albuñol, dans le sud des Alpujarras de la province de Grenade en 1944, Gabriel Amat fait partie de ces familles d'agriculteurs qui migrèrent en provenance des Alpujarras.

\section{Conclusion}

À l'échelle locale, une économie entière peut reposer essentiellement sur la main-d'œuvre immigrée comme le montre le cas du Poniente almeriense. Les élus locaux, s'ils ne font rien pour favoriser l'intégration des travailleurs immigrés, sont bien conscients de l'intérêt qu'ils ont à avoir une maind'œuvre abondante et bon marché sur leur territoire afin de maintenir la rentabilité du secteur le plus important de l'économie locale. Depuis les émeutes de 2000 à El Ejido, les propos anti-immigration y sont donc devenus très rares.

À l'inverse, à l'échelle nationale et européenne, l'immigration irrégulière continue d'être l'objet d'une stigmatisation et de politiques de contrôle toujours plus strictes (notamment à travers une externalisation du contrôle des frontières). Cette contradiction entre le local et l'international fait d'un territoire comme le Poniente almeriense un lieu de tensions potentielles, particulièrement en situation de crise économique. 